\title{
The effect of orientation on interpolated elastic structure from dot motion: Its occurrence and persistence
}

\author{
AKIRA ISHIGUCHI \\ University of Tokyo, Tokyo, Japan
}

\begin{abstract}
The aim of this study was to investigate properties of an interpolation process by which the human visual system interprets the structure of an object from motion. Two dots moving in a plane were used as a stimulus display. One dot was fixed at the center of the display, and the other moved part way around the fixed dot like an endpoint of a rigid pendulum. Subjects were asked to categorize the structure of an object perceived from the motion of the dots. In Experiment 1 , the orientation of the symmetry axis of the simulated pendular motion was varied. The results showed that the structure was perceived to be elastic and bending, not rigid. Furthermore, the perceived structure was affected differently by the orientation of the symmetry axis of motion. In Experiment 2, the time-course change of this effect was studied. The results showed that both the orientation of the symmetry axis of motion and the position and orientation given at the initial stage of motion influenced the structure perceived from the dot motion, and their influences changed over time. These results suggest that position- and orientation-extraction mechanisms are involved in the process of the perception of structure from motion.
\end{abstract}

The capacity of the human visual system to interpret the three-dimensional (3-D) structure of viewed objects from the motion of their elements projected in a frontal plane has been studied by many researchers (Braunstein, 1962, 1976; Green, 1961; Hoffman \& Flinchbaugh, 1982; Johansson, 1973, 1975; Todd, 1982; Ullman, 1979, 1984; Wallach \& O'Connell, 1953). The main problem posed by many of these studies is the ambiguity of the interpretation. To solve this ambiguity, some researchers have suggested that a rigidity constraint must be incorporated in the interpretation scheme (see Uliman, 1983, for a review), but others have not needed the rigidity assumption to account for their results (Bennet \& Hoffman, 1985).

Despite the different views on the rigidity assumption, many researchers have had a tacit understanding that 3D structures can be recovered from motion with some additional constraints if the position of elements in the image plane can be extracted accurately. In line with this understanding, some researchers have attempted theoretically to find out the minimum information needed for recovery of structure from motion (Bennet \& Hoffman, 1985; Hoffman \& Flinchbaugh, 1982; Ullman, 1979). On the other hand, Ishiguchi (1988) showed empirically that Hoffman and Flinchbaugh's (1982) scheme of recovery

This research was supported in part by a Grant-in-Aid for Encouragement of Young Scientists, Ministry of Education, Science and Culture, No. 62710034. The author wishes to thank J. M. Foley of the University of California, Santa Barbara, for his helpful comments on an earlier version of this article. Requests for reprints should be sent to Akira Ishiguchi, Faculty of Letters and Educations, Ochanomizu University, 2-1-1 Otsuka, Bunkyo-ku, Tokyo 112, Japan. of a rigid rod from the motion of its two elements was inadequate for the human visual system. Ishiguchi (1988), using dots in motion, demonstrated the rubber pencil illusion reported by Pomerantz (1983). The rubber pencil illusion is a bending appearance of a physically rigid straight pencil that is "easily produced by wiggling a pencil that is held loosely and off-center between the thumb and index finger" (Pomerantz, 1983, p. 365). Ishiguchi (1988) simulated the motion of two endpoints of a wiggling rigid pencil with the motion of two dots, and obtained the results that observers perceived an elastic rod from the motion of the dots. The elastic structure was perceived also under the condition in which the actual 3-D plane where the dots moved (not the projection plane) tilted in depth, that is, under the conditions that produced the kinetic depth effect. Furthermore, Ishiguchi (1988) gained counterevidence to the possibility that the perception of this elastic structure resulted from the extraction of information intrinsic to the motion of an elastic body.

The experiments reported here were conducted to elucidate the visual mechanism producing the elastic structure from the motion of dots that act as endpoints of a physically rigid rod.

\section{EXPERIMENT 1}

In this experiment, the motion of two endpoints of a rigid pendulum was displayed. Rigidity in this study means that the Euclidean distance between two dots in motion is preserved. Ishiguchi (1988) showed that an elastic percept, such as the rubber pencil illusion, can be obtained from the motion of two dots even when one dot is stationary. Since the purpose of this experiment was to ex- 
amine the effect of orientation on this perceived elastic structure, the simple stimulus display that was used had one endpoint of a simulated rigid pendulum fixed and stationary, and the other oscillating around the fixed endpoint. In this experiment, the orientation of the symmetry axis of the pendular motion was varied.

\section{Method}

Subjects. Twelve students (8 undergraduates and 4 graduates) participated in this experiment. All students except one had participated in preceding experiments on this phenomenon. They all had normal or corrected-to-normal vision.

Apparatus. Stimulus patterns were generated by a microcomputer (NEC PC-9801m) and presented on a CRT (KIKUSUI COS 1711 with a $\mathrm{p} 31$ phosphor), which was placed at a distance of $57.5 \mathrm{~cm}$ from the eyes of the subjects. The subjects sat in a chair with a chinrest and observed the CRT binocularly. The room was completely dark.

Stimulus and Procedure. The stimulus display consisted of two dots that simulated the endpoints of a physically rigid pendulum. In an actual display (e.g., see Figure 1), one dot (D1 in Figure 1) was fixed at the center of the display, and the other dot (D2 in Figure 1) oscillated pendulously around D1. The amplitude of the pendular oscillation was kept constant at $30^{\circ}$, and the frequency was $1 \mathrm{~Hz}$. This oscillation lasted $5 \mathrm{sec}$. As a result, the oscillation repeated five times. The distance of the two dots was $3^{\circ}$ in visual angle, and the luminance of the dots was $7.8 \mathrm{~cd} / \mathrm{m}^{2}$.

The experimental variable was the orientation ( $\theta$ in Figure 1 ) of the symmetry axis of the pendular ascillation. This axis corresponded to an imaginary line (e.g., the dotted and broken line in Figure 1) passing from D1 through the center of the trajectory of D2. Of course, this line was not actually drawn on the screen. The orientation was varied from $0^{\circ}$ to $345^{\circ}$ in $15^{\circ}$ steps, which resulted in a total of 24 displays. Each display was presented three times in random order. The oscillation of a simulated rigid pendulum started counterclockwise from the position of the symmetry axis (e.g., the position indicated by closed circles in Figure 1).

The subjects were required to categorize the structure perceived from the motion of the dots as follows: 0-The two dots appear to constitute no structure. 1-The two dots appear to constitute a rigid structure (see Figure 2a). The following three category num-

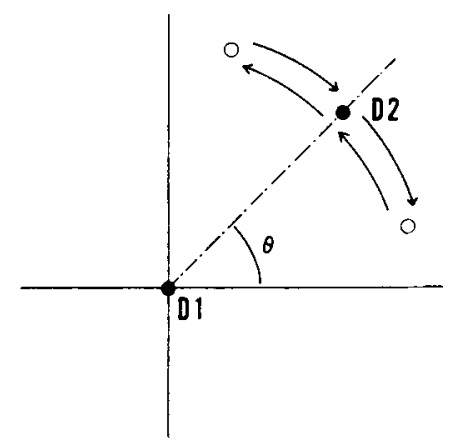

Figure 1. One example of the stimulus configuration used in Experiment 1. D1 is a dot stationary at the center of the display, and D2 is a dot that moves like an endpoint of a pendulum around D1. Dotted and broken line is the symmetry axis of motion of D2, and $\theta$ is the orientation value of the axis from the horizontal. The pendular oscillation started counterclockwise. The closed circles indicate the first position of the dots when they started to move.
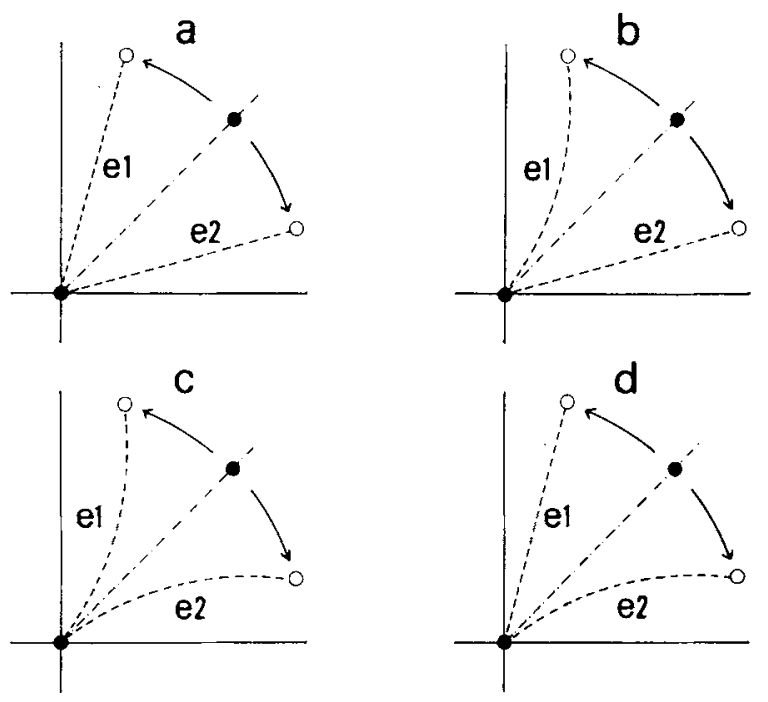

Figure 2. Four typical examples of perceived structure (see text for category descriptions): (a) rigid structure corresponding to the category number 1, (b) asymmetric elastic structure corresponding to the category number 21 , (c) symmetric elastic structure corresponding to the category number 22 , and (d) asymmetric elastic structure corresponding to the category number 23 . The lines e1 and 2 indicate a momentarily perceived structure.

bers were chosen when two dots appeared to constitute an elastic and bending structure:

21-The perceived structure is asymmetric (Figure 2b) such that the structure is perceived to be more elastic when the orientation angle of $\overline{D 1 D 2}$ is larger than the symmetry axis, than when the orientation angle of $\overline{\mathrm{D} I D 2}$ is smaller than the symmetry axis. That is, in Figure $2 \mathrm{~b}$, the momentarily perceived line $\mathrm{el}$ is more elastic than $\mathrm{e} 2$.

22-The perceived structure is elastic symmetrically to the symmetry axis (Figure 2c).

23-The perceived structure is asymmetric (Figure 2d) such that the structure is perceived to be more elastic when the orientation angle of $\overline{D 1 D 2}$ is smalier than the symmetry axis, than when the orientation angle of $\overline{\mathrm{D} 1 \mathrm{D} 2}$ is larger than the symmetry axis. That is, in Figure $2 d$, the momentarily perceived line 2 is more elastic than $\mathrm{el}$.

The subjects were instructed to fixate their eyes at the center of the display (i.e., at the position of D1). The subjects performed 72 test trials after 10 practice trials.

\section{Results}

In the results of Ishiguchi (1988), about $10 \%$ of reported category numbers were 0 under the condition in which D1 was stationary. However, in this experiment, no category number 0 was reported; that is, the subjects always observed some structure from the motion of the dots in every display. This difference is probably because the distance of the two dots was $3^{\circ}$ in visual angle in this experiment, but $5^{\circ}$ in Ishiguchi's (1988) study. Under the condition in which both dots oscillated, some structure created by the movement of the dots was almost always perceived, even when the distance of the two dots was $6^{\circ}$ in visual angle (Ishiguchi, 1988). It is possible that reti- 
nal eccentricity has more effects on interpolation of structure from motion when one dot is stationary than when both dots move.

Figure 3 shows the total frequencies of each category number reported by all subjects as a function of the orientation of the symmetry axis. Since the 12 subjects observed each stimulus display three times, the sum of the frequencies of category numbers in each orientation of the axis came to 36. The thick solid line in Figure 3 indicates the frequency of category number 22 : the perceived structure is symmetrically elastic in relation to the symmetry axis of motion. The thick dotted line indicates the frequency of category number 21 , and the thin dotted line indicates the frequency of category number 23 . The frequency data of the category number 1 are removed from this figure due to its few frequencies. Figure 3 shows the large effect of the orientation of the symmetry axis, in particular, the effect of the horizontal or the vertical axis (i.e., the principal meridians) on the appearance of the perceived structure: When the orientation of the symmetry axis was horizontal or vertical, category number 22 was frequently reported. On the other hand, when the orientation angle of the axis was larger by $15^{\circ}$ or $30^{\circ}$ counterclockwise than that of the horizontal or the vertical axis, category number 21 was often reported. In the same way, when the orientation angle of the axis was smaller by $15^{\circ}$ or $30^{\circ}$ than that of the horizontal or the vertical axis, category number 23 was frequently reported. These results can be interpreted as follows: the perceived structure of a rod constituted by dots appeared more elastic as the rod moved away from the horizontal or the vertical axis.

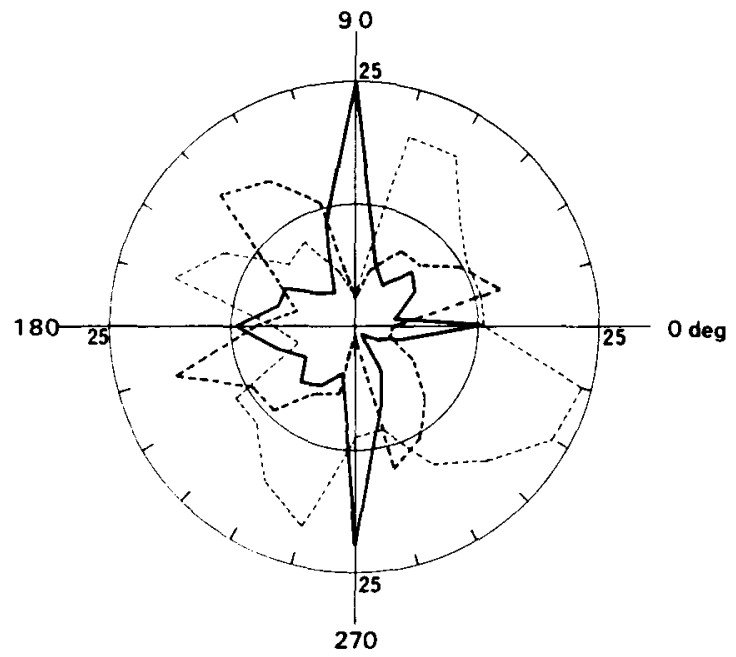

Figure 3. Total frequencies of each category number as a function of the orientation of the symmetry axis. The thick solid line indicates the frequency of category number 22 , the thick dotted line indicates the frequency of category number 21 , and the thin dotted line indicates the frequency of category number 23 . The line indicating the frequency of category number 1 was removed from this figure due to its few frequencies.
Table 1

Computational Procedure to Transform Raw Frequencies of the Reported Category Number in Each Orientation of Symmetry Axis of Motion into the Vahue Indicating the Degree of Effect on the Perceived Structure

\begin{tabular}{cccccc} 
& \multicolumn{5}{c}{ Orientation Angles of Axes } \\
\cline { 2 - 6 } Category Number & $\theta-30^{\circ}$ & $\theta-15^{\circ}$ & $\theta$ & $\theta+15^{\circ}$ & $\theta+30^{\circ}$ \\
\hline 1 & 1 & & 1 & & 1 \\
21 & 2 & 1 & 3 & & \\
23 & & & 3 & 1 & 2 \\
\hline
\end{tabular}

Note-Under any condition in which the orientation angle of the symmetry axis is $\theta$, three points were allotted, according to the reported category number.

To clarify directly the effect of the orientation of the symmetry axis, as shown in Figure 3, the frequencies of the reported category numbers were transformed into the values indicating this effect. The computational procedure of the transformation is summarized in Table 1 . The essential point is that when a category number is reported under the condition of the orientation $(\theta)$ of the symmetry axis, some weights, the total of which is three for each response, are allotted among a set of orientations $(\theta$, $\theta \pm 15^{\circ}$, and $\theta \pm 30^{\circ}$ ) of some symmetry axes, including the orientation of the symmetry axis concerned and those used under other conditions. The values of $15^{\circ}$ and $30^{\circ}$ resulted from the procedure of this experiment, in that the amplitude of the pendular oscillation was constant at $30^{\circ}$ and the orientation of the symmetry axis was varied in $15^{\circ}$ steps. As shown in Table 1, reported category numbers determine how these weights are allotted, based on the following considerations: It was found that the structure of a rod perceived from the motion of dots came to be more elastic as the rod moved away from orientations of some axes. Thus, it can be thought that these orientations of the axes have strong effects on the perceived elastic structure. This is why the weights, corresponding to the degree of the influence on the perceived elastic structure, were given to the orientation of the axis. For example, when category number 21 was reported under the condition in which the orientation $(\theta)$ of the symmetry axis was $120^{\circ}$, two weights were allotted to the orientation of the $90^{\circ}\left(\theta-30^{\circ}\right)$ axis, and one weight, to the orientation of the $105^{\circ}\left(\theta-15^{\circ}\right)$ axis. This is because the perceived structure corresponding to the category number $\mathbf{2 1}$ came to be more elastic as it moved away from the orientation of the $90^{\circ}$ axis.

This scoring procedure resulted in Figure 4, in which the rates of the scores allotted to the orientations of the symmetry axis of motion are plotted. These rates are values of the scores of each orientation of the axis to the score of the axis with the $90^{\circ}$ orientation. This figure shows directly the different effects of the symmetry axis, in particular, the predominant effect of the principal meridians, or of the orientation of the horizontal or the vertical axis. 


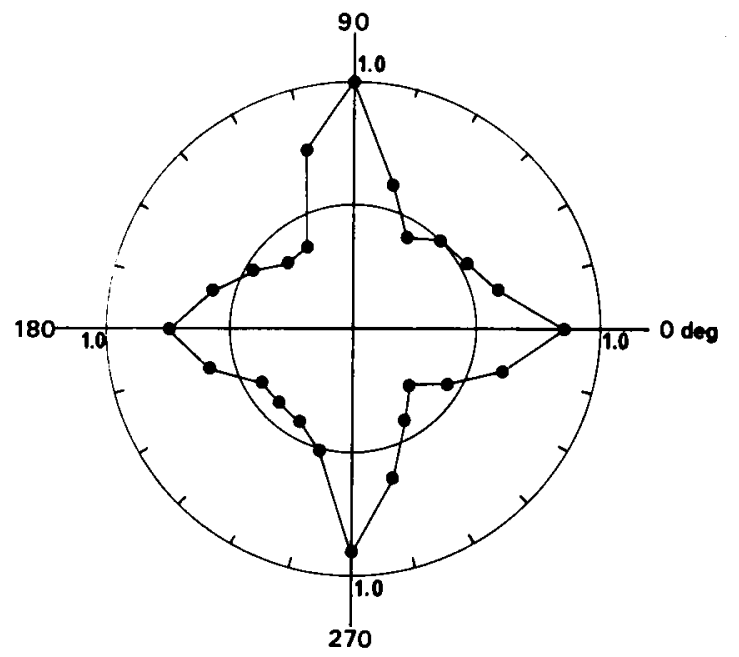

Figure 4. The effect of orientation of exch symmetry axis on the perceived structure. The values of each orientation were obtained with the computational procedure in Table 1 using the frequencies of category numbers in each orientation in Figure 3. Furthermore, these values were rated to the value of $90^{\circ}$ orientation.

\section{Discussion}

These results can be summarized as follows:

1. A rod was perceived from the motion of the two dots that corresponded to the two endpoints of a rigid pendulum, one of which was fixed.

2. The perceived structure of the rod was often elastic and bending, whereas the simulated structure was rigid.

These results confirmed the results of Ishiguchi (1988). Although the motion of the two endpoints of a rigid pendulum was simulated, an elastic and bending pendulum, not a rigid one, was often observed from the motion of the points. This simulated rigidity was based on the fact that the distance of the elements of the rigid body is always kept constant when the rigid body moves in a frontal parallel plane. The reverse of this fact is not always true; that is, when an elastic body moves in the frontal plane, the distance of its two elements may be kept constant. However, in this latter case, our visual system cannot recover uniquely the elastic structure unless it extracts more information about the elastic body, such as a phase difference between motions of the elements. As shown by Ishiguchi (1988), however, this percept, the elastic rod, was not due to the extraction of the information intrinsic to the motion of an elastic body: the phase difference between the motions of the elements and the changing distance between the elements. Thus, it is probable that other mechanisms-not the mechanism of the recovery of structure from motion, the algorithm for which has been developed by many computational approaches-were involved. Other results in this experiment also suggest other mechanisms.

3. The orientation of the symmetry axis of motion of the dots-in particular, that of the horizontal or the vertical symmetry axis-had different strong effects on the perceived structure from the motion of the dots. As the perceived rod moved away from the principal meridians, if the symmetry axis was near the principal meridians, the structure was perceived as being more like an elastic rod. Rigidity was perceived near the principal meridians.

As mentioned above, the perceived moving rod did not always bend symmetrically. In Experiment 1, the perceived structure was influenced differently by the orientation of the symmetry axis of motion. These results suggest that the perceived structure is not only determined by the information about the position of the dots in the 2-D coordinate plane. In addition to a mechanism extracting the position of the dots, it is probable that a mechanism extracting the orientation of the rod consisting of the dots is involved. The orientation-extraction mechanism means that, when two (or more) dots move, the orientation of a momentary interpolated line between the dots will be extracted. The involvement of the orientationextraction mechanism can explain the results that the horizontal or the vertical orientation had a predominant effect on the perceived structure.

If the position- and orientation-extraction mechanisms are involved, how do they operate when the human visual system constitutes a structure from the motion of dots? Based on the results of Experiment 1, it may be thought that these two mechanisms operate simultaneously on the information about the moving dots, and their outputs may be integrated in a higher interpolation process to produce a subjective rod connecting the dots. It may be hypothesized that the amount of the outputs from the two mechanisms determines the degree of persistence strength of the structure of the subjective rod. Although many subjective rods are produced during the motion, their structure may be influenced by the subjective rod with a higher degree of persistence strength. This process may account for the results that, as the perceived rod moved away from the principal meridians, its structure was perceived to be more like an elastic rod. The output from the orientationextraction mechanism may increase when the orientation of the two dots corresponds to the principal meridians. Thus, the subjective rod with the orientation of the principal meridians will have a higher degree of persistence strength. The structures of rods with other orientations of the principal meridians will be influenced by the rod with a high degree of persistence strength, and those structures will be perceived as bending, not rigid. This persistence effect, however, may not be similar to the persistence process that is the basis of the rubber pencil illusion (Pomerantz, 1983), because the latter is concerned with the total trajectory of a moving pencil as it persists in an early visual system.

The aim of Experiment 2 was to examine the assumed process mentioned above in more detail, and to investigate the property of information that the human visual system extracts in the process of constituting the structure from the motion of dots.

\section{EXPERIMENT 2}

In Experiment 1, it was found that the orientation of the symmetry axis of motion of dots has different in- 
fluences on the perceived structure of a moving rod. Experiment 2 was carried out not only to examine whether these influences would change over time, but also to examine the possibility that other variables exist that have some effects on the perceived structure.

\section{Method}

Subjects. Ten students ( 6 graduates and 4 undergraduates) participated in this experiment. Five of these students had not served in Experiment 1; the other 5 had been in Experiment 1. All had normal or corrected-to-normal vision.

Apparatus. The apparatus was the same as in Experiment 1.

Stimulus and Procedure. Each stimulus consisted of two dots, as in Experiment 1. One dot was stationary at the center of the display, and the other moved like one endpoint of a pendulum. The main variable used in Experiment 1 was the orientation of the symmetry axis of the dot motion. In this experiment, three main variables were used. The first variable was the initial stationary period, the duration during which the two dots were stationary before they began to move. This period was 0,2 , or $4 \mathrm{sec}$. The second variable was the number of oscillation repetitions. In Experiment 1 , the repetition number was kept constant at 5 . In this experiment, the repetition number was $1,2,4$, or 8 . The third variable was the orientation of the symmetry axis of motion. This orientation was varied from $-30^{\circ}$ to $210^{\circ}$ in $15^{\circ}$ steps, where $0^{\circ}$ corresponds to a horizontal orientation with the moving point to the right of the stationary point. In addition to these three main variables, two initial directions of pendular motion were used; that is, the pendular motion started clockwise or counterclockwise. In Experiment 1, the dot always started to move counterclockwise from the position on the symmetry axis of motion. In Experiment 2, the dot started to move from the position of the outermost part of the trajectory (Figure 5). Thus, 408 stimulus conditions were presented (3 stationary periods $\times 4$ oscillation repetitions $\times 17$ orientations $\times 2$ initial directions), each three times.

The experiment consisted of eight sessions, each performed on a different day. One session consisted of 153 trials: with both the repetition number of motion and the initial direction of motion fixed, three initial stationary periods were combined with 17 orientations of the symmetry axis, and each was presented three times in random order.

The procedure of the experiment was the same as in Experiment 1 except that the subjects were additionally required to categorize the structure perceived from the dot motion at the last cycle of repeated motion, so that the effect of the repetition on the perceived structure could be examined more clearly. The test trials followed $\mathbf{1 0}$ practice trials in each session.

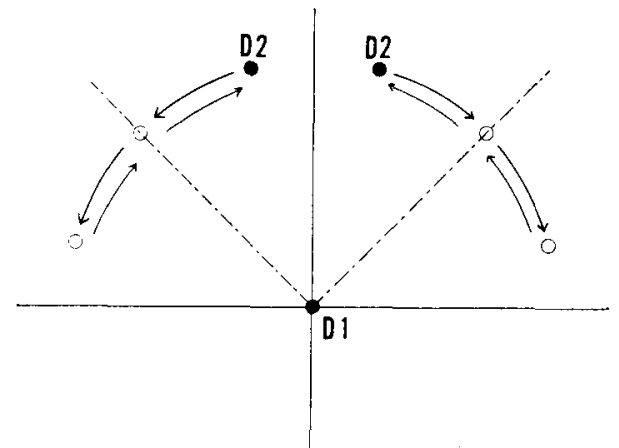

Figure 5. Examples of stimulus configuration used in Experiment 2. A moving dot D2 started from the position of the outermost part of the trajectory, unlike in Experiment 1, and moved clockwise or counterclockwise.

\section{Results and Discussion}

Among the reported category numbers, the category number 0 , which meant that the two dots appeared to move separately, was reported as infrequently as in Experiment 1 . Of the 1,530 responses made by the 10 subjects in each session, the maximum number of the category 0 reported was 10 , and the average was 7.3. Apparently, the subjects perceived a line interpolated between the dots under every condition.

Next, I examine the effect of the variables used in this experiment on the structure perceived from the dot motion. Three main variables were used in this experiment. The first was the orientation of the symmetry axis of motion, which varied from $-30^{\circ}$ to $210^{\circ}$ in $15^{\circ}$ steps. The second variable was the number of repetitions of a pendular oscillation. This variable was used to examine the time-course variation, not only of the different effects of the orientation of the symmetry axis obtained in Experiment 1 , but also of the effect of the position and the orientation given at the initial stage of motion. The third variable was the initial stationary period before the dots started to move. This variable was used to examine the hypothesis that the persistence strength of a subjective line, with the position and the orientation given at the initial stage, has some influences on the perceived structure from the dot motion. If so, the stationary periods at the initial stage of motion will have different effects on the perceived structure.

I examined the effect of the orientation of the symmetry axis on the perceived structure, when both the number of repetitions of the pendular oscillation and the initial stationary period were also varied. The values in Figure 6 are those transformed according to the procedure given in Table 1 in order to clarify the effect, as done in Figure 4. In Figure 6, the number of repetitions (NR) of the pendular oscillation increases from left to right, and the initial stationary period (ISP) increases from top to bottom. As shown in Figure 6, when the dots oscillated only one cycle, orientations of the symmetry axis had equal influences on the perceived structure, and the predominant effect of the vertical or the horizontal orientation that was obtained in Experiment 1 was not again obtained. When the motion repeated twice under the condition in which the initial stationary period was $0 \mathrm{sec}$, that effect appeared vividly, but it did not under the condition in which the initial stationary period was 2 or $4 \mathrm{sec}$. On the other hand, with four or eight repetitions, the effect of the horizontal and, in particular, of the vertical orientation manifested itself regardless of the length of the initial stationary period.

Figure 7 shows both the effect of the number of oscillation repetitions and the effect of the initial stationary period on the structure perceived from the dot motion. The reported category numbers on each orientation of the symmetry axis of motion were pooled. The ordinate represents the proportion of the following pooled structures reported by subjects: "Bending Structure A" means that the structure was perceived as bending less when the orientation of the perceived rod corresponded to the orien- 


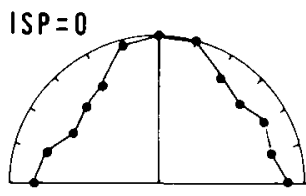

ISP $=2$
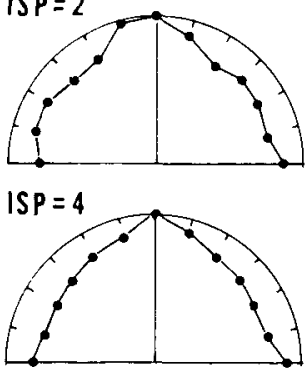

$N R=1$
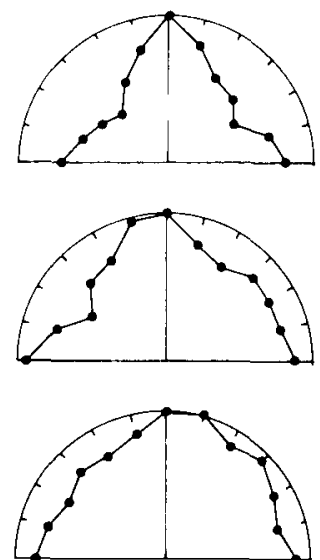

$N R=2$
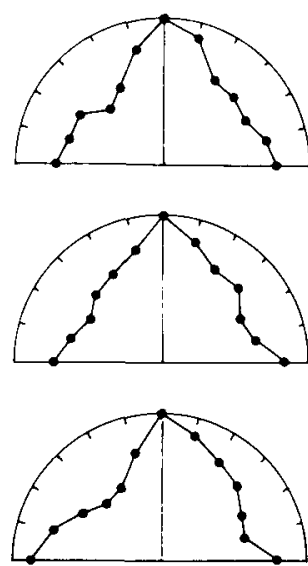

$N R=4$
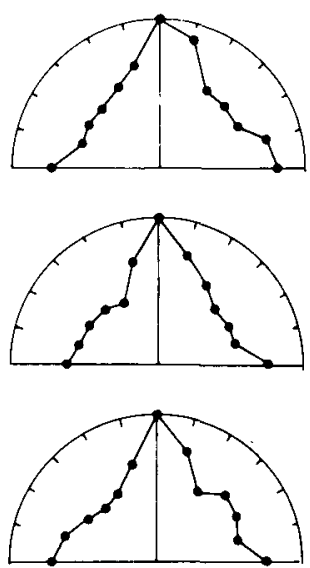

$N R=8$

Figure 6. The effect of the orientation of each symmetry axis on perceived structure. These values were obtained using the same procedure used for the values in Figure 4. The number of oscillation repetitions (NR) is increased from the left to the right columns, and the initial stationary period (ISP) is increased from the top to the bottom rows.

tation given at the initial stage of motion (Figure 8a) than when the perceived rod moved to the opposite side of the symmetry axis. The category number corresponding to Bending Structure A is 23 when the initial direction of the pendular oscillation was clockwise and 21 when it was counterclockwise. Thus, the proportion of Bending Structure A perceptions indicates the persistent effect of the position and the orientation given at the initial stage of motion. "Bending Structure B"' (Figure 8b) means the reversed appearance of the Bending Structure A. "Bending Structure C"' (Figure 8c) means that the structures, which are opposite sides of the symmetry axis of motion, are perceived as bending similarly. The category num- ber corresponding to the Bending Structure $\mathrm{C}$ is 22 in every condition. "Rigid Structure" (Figure 8d) corresponds to the category number 1 .

I analyzed the effects of the number of oscillation repetitions and the initial stationary period on the perceived structure. First, the effect of the number of oscillation repetitions was analyzed. The proportion of Bending Structure A perceptions reported by the subjects decreased as the number of oscillation repetitions increased. This result suggests that the effect of the position and the orientation given at the first stage of motion fades out with an increased number of oscillation repetitions. In addition, it is very important that the proportion of Rigid Structure

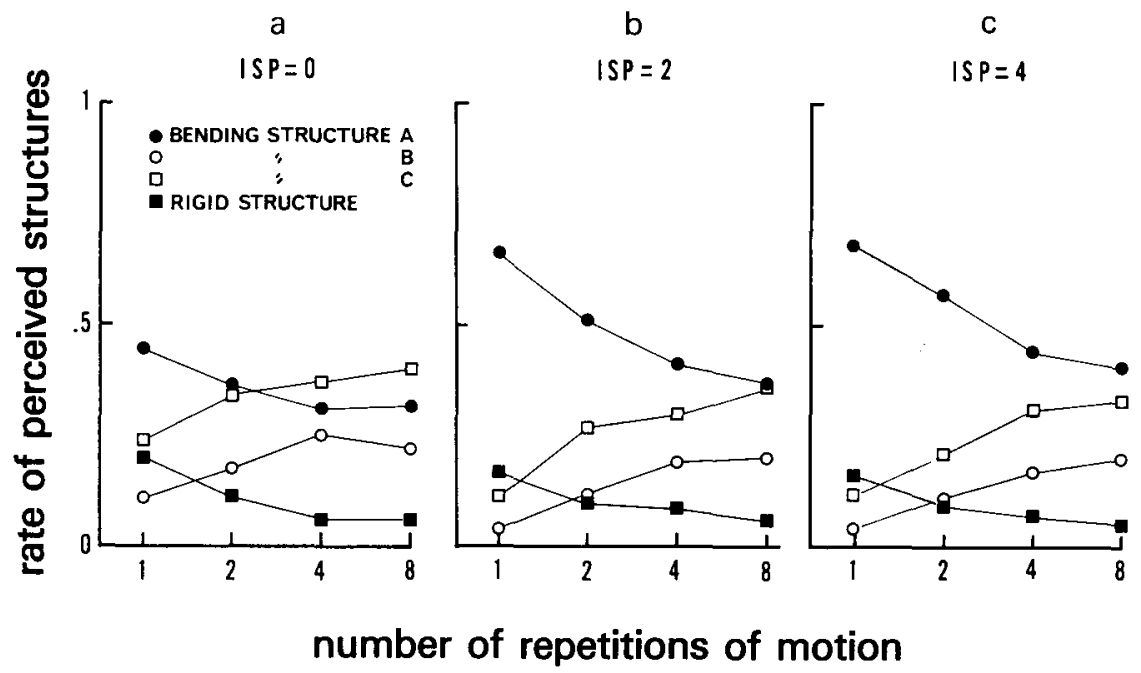

Figure 7. The proportion of perceived structures as a function of the number of oscillation repetitions, under the conditions in which the initial stationary period (ISP) was $0 \mathrm{sec}$ (a), $2 \sec (b)$, or $4 \sec ($ c). 
a

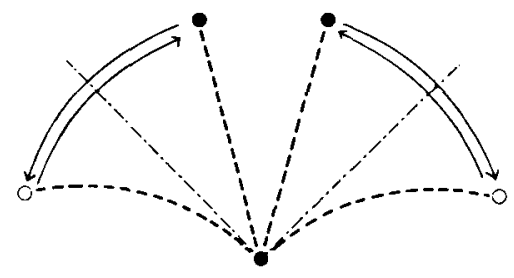

C

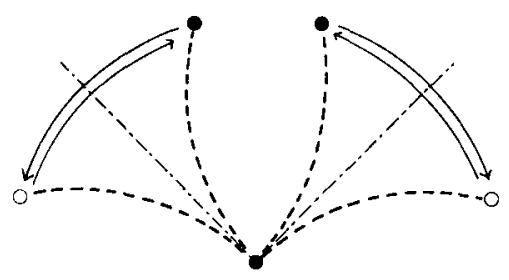

b
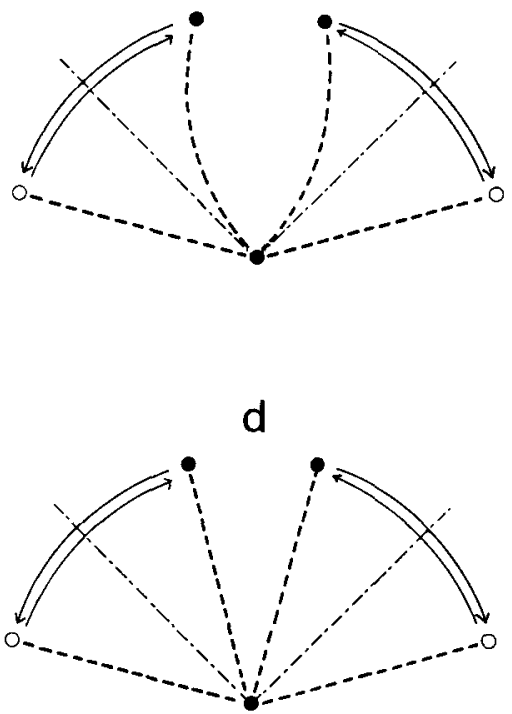

Figure 8. Typical examples of the structures perceived from the motion of the dots. The frequencies of these four kinds of categorical structures obtained in Experiment 2 were used to calculate the rates in Figure 7 .

responses decreased with an increase in the number of repetitions. This result represents evidence that counters Ullman's (1984) hypothesis that a rigid structure is recovered gradually from motion (i.e., the incremental recovery of structure assumption). The results of this experiment show that an elastic structure, not the simulated rigid structure, may be perceived as the motion repeats.

In contrast to perceptions of both the Bending Structure $A$ and the Rigid Structure, Bending Structure B and Bending Structure C perceptions increased with an increase in the number of oscillation repetitions. It is particularly important that the proportion of Bending Structure $\mathbf{C}$ perceptions increased. Since Bending Structure $\mathrm{C}$ refers to a structure that is perceived to be symmetrically bending, the increase in the proportion of perceptions of this structure indicates that the orientation of not only the principal meridians, but also of each symmetry axis of motion, comes to have an effect on the perceived structure with an increase in the number of oscillation repetitions.

Second, the effect of the initial stationary period was analyzed. Even when the initial stationary period was $0 \mathrm{sec}$, a higher proportion of Bending Structure A perceptions was obtained than any other perceived structure under the condition in which the pendular oscillation repeated once. In the stimulus display used in this experiment, the velocity was lowest at the initial stage of motion because of the property of the simple pendular oscillation. Thus, if both the position- and the orientationextraction mechanisms operate instantaneously with the presentation of the stimulus, it is possible that the position and orientation of the perceived rod with the lowest velocity at the first stage of motion are extracted and consequently have some persistent effects on the perceived structure. Furthermore, these data in Experiment 2 suggest that the effect of the position and orientation given at the initial stage of motion is stronger at the early stage of motion than is the effect of the orientation of the symmetry axis. However, the former effect decreases and the latter effects increase as the motion repeats. The time the human visual system requires to manifest the different effects of the symmetry axis depends on the length of the initial stationary period. In addition, it should be noted that the effect of the initial stationary period is nonlinear. As shown in Figure 6, the 2-sec initial stationary period has a larger persistent effect on the perceived structure than does the 0-sec initial stationary period, but it has a similar effect to the 4-sec initial stationary period. This is confirmed in Figure 7, which indicates that the proportion of Bending Structure A perceptions increased nonlinearly with the increase in the length of the initial stationary period.

\section{GENERAL DISCUSSION}

As shown by the results of these two experiments, the structure of the rod perceived from the motion of dots is influenced by several variables: the orientation of the symmetry axis, the number of oscillation repetitions, and the length of the initial stationary period.

When researchers have examined the ability of the human visual system to recover the 3-D structure from 2-D motion projected in the frontal parallel plane, they have considered only a position-extraction mechanism and its algorithm. In this case, major problems are what amount of information is necessary to recover a unique structure (Bennet \& Hoffman, 1985; Hoffman \& Bennet, 1986; Hoffman \& Flinchbaugh, 1982; Tsai \& Huang, 1981; 
Ullman, 1979) and whether the rigidity assumption applies (Gibson \& Gibson, 1957; Green, 1961; Johansson, 1975; Ullman, 1979; Wallach \& O'Connell, 1953). The latter problem has been raised because some constraints are necessary in order to solve the ill-posed problem of recovering 3-D structure from 2-D projected image. However, based on the results reported here, it appears that such approaches have overlooked a basic mechanism of the human visual system. Ishiguchi (1988) and the present experiments showed that the perceived structure from motion was elastic and bending, not rigid, even when the visual system extracted the position of moving dots corresponding to the endpoints of a rigid rod. This perception was not due to the process in which the human visual system extracts the information intrinsic to the motion of the elastic body (Ishiguchi, 1988); that is, the position-extraction mechanism alone cannot sufficiently explain the perception of structure from motion.

Based on the results of the present experiments, it is necessary to consider the properties of an interpolation process that includes an orientation-extraction mechanism in the perception of structure from motion. The results of Experiment 2 showed that when two dots oscillated like two endpoints of a pendulum after the two dots had been seen as stationary for some seconds, the position and orientation of the dots at the initial stage of motion had a strong effect on the subsequent perceived structure. However, this effect decreased as the motion continued. Instead of the initial position and orientation, then, the orientation of the symmetry axis of motion, in particular that of the principal meridians, came to affect the perceived structure from motion as the number of oscillation repetitions increased. Thus, both effects might reflect the properties of processes by which the interpolation mechanism operates.

These data also suggest that the position- and the orientation-extracting mechanisms operate simultaneously in the interpolation process, and that the degree of the persistence strength of interpolated lines may be determined by the amount of the outputs from these two mechanisms. It is possible to consider that certain initial stationary periods increase the outputs of the two mechanisms, which results in strengthening the degree of persistence of the subjective rod with the position and orientation given at the initial stage. This consideration may be confirmed by the results that the effect of the initial stationary period faded out over time. Furthermore, the results showed that the perceived structure was influenced predominantly by the vertical or the horizontal orientation in the display in which the moving stimulus was presented. The subjects reported that the structure of the rod was perceived as more bending when the perceived rod passed away from the principal meridians. These results also suggest that the orientation-extraction mechanism operates in this process. In this respect, there are neural-based suggestions that the vertical or the horizontal lines are detected more preferentially than the oblique lines (Appelle, 1972), and that this oblique effect may be caused by an anisotropy in the higher order mechanism (Vogels \& Orban, 1985). Thus, the outputs from the orientation-extraction mechanism may increase when the orientation of the dots corresponds to the vertical or the horizontal orientation, and the subjective rod with such orientations appears rigid.

The interpolation process mentioned above may solve one of the problems discussed by Ishiguchi (1988): Why does an increase in the number of dots increase the perceived rigidity? In all existing relevant electrophysiological works on orientation selectivity of visual cortical cells, only a single line has been used as the stimulus. However, psychophysical data show that separating the dots triggers the same orientation-detecting processes as do the line segments (Bouma \& Andriessen, 1968; Brown \& Kortela, 1977). Thus, it is possible that if the number of dots increases, the position and orientation of the rod consisting of the dots will be detected with a higher degree in each motion stage, which is partly due to the vernierlike mechanism. In this situation, a variable such as the orientation of the symmetry axis, the number of oscillation repetitions, or the length of the initial stationary period has less influence on the perceived structure, and a simulated rigid structure can be perceived. In this connection, the relation to the mechanism that produces the rubber pencil illusion (Pomerantz, 1983) should be discussed. A wiggling physically rigid line was perceived to be elastic. Pomerantz (1983) suggested that this phenomenon is due to the visual persistence occurring at the early stage (perhaps in the retina) of the visual process. On the other hand, my unpublished data showed that the elastic structure from the motion of dots was perceived even under a dioptic viewing condition. In addition, Ishiguchi (1988) showed that the elastic structure from the motion of dots was perceived vividly, even under the condition in which the dots oscillated at $0.2 \mathrm{~Hz}$. On the other hand, the rubber pencil illusion diminished at such a low frequency (the strength of the illusion was maximized at $3.2 \mathrm{~Hz}$; Pomerantz, 1983). Thus, it is probable that the mechanism producing the elastic structure observed in the experiment reported here is different from the mechanism producing the rubber pencil illusion.

However, another problem may remain unsolved: Why does the phase difference between the motions of the dots affect the degree of elasticity perceived from the dots' motion (Ishiguchi, 1988)? In the experiments reported here, since one dot was stationary at the center of the display, there existed no phase difference between the motions of the dots. Thus, it was reconfirmed that the information about the phase difference cannot serve as the causal input to the process that produces the elastic structure. Ishiguchi (1988) showed, however, that the elasticity of the structure perceived from the motions of dots varied with the variation of the phase difference between the motions of dots. The hypothesized interpolation mechanism in the present study may not be involved in the difference of elasticity induced by the phase difference; therefore, it is possible that the process that produces a struc- 
ture from motion includes other mechanisms in addition to the position-extraction mechanism, the orientationextraction mechanism, and the integrating mechanism operating on outputs from the formers. In addition, researchers should look into how the local elasticity reported here is organized into a global rigidity as shown by the biomechanical motion (Bertenthal, Proffitt, \& Kramer, 1987; Cutting \& Proffitt, 1981).

\section{REFERENCES}

APPELle, S. (1972). Perception and discrimination as a function of stimulus orientation: The oblique effect in man and animals. Psychological Bulletin, 78, 266-278.

BenNet, B. M., \& HofFMAN, D. D. (1985). The computation of structure from fixed-axis motion: Nonrigid structures. Biological Cybernetics, 51, 293-300.

Bertenthal, B. I., Proffitt, D. R., \& Kramer, S. J. (1987). Perception of biomechanical motions by infants: Implementation of various processing constraints. Journal of Experimental Psychology: Human Perception \& Performance, 13, 577-585.

Bouma, H., ANDRIESSEN, J. J. (1968). Perceived orientation of isolated line segments. Vision Research, 8, 493-507.

Braunstein, M. L. (1962). Depth perception in rotation dot patterns: Effect of numerosity and perspective. Joumal of Experimental Psychology, 64, 415-420.

Braunstein, M. L. (1976). Depth perception through motion. New York: Academic Press.

Brown, J. L., Kortela, I. M. (1977). Orientation discrimination. In S. J. Cool \& E. L. Smith (Eds.), Frontiers in visual science (pp. 286-297). New York: Springer-Verlag.

CutTing, J. E., \& ProffitT, D. R. (1981). Gait perception as an example of how we may perceive events. In R. Walk \& H. L. Pick (Eds.) Intersensory perception and sensory integration (pp. 249-273). New York: Plenum Press.

Gibson, J. J., \& Girson, E. J. (1957). Continuous perspective transformations and the perception of rigid motion. Joumal of Experimental Psychology, 54, 129-138.
GreEN, B. F. (1961). Figure coherence in the kinetic depth effect. Journal of Experimental Psychology, 62, 272-282.

HoFfman, D. D., \& BENNET, B. M. (1986). The computation of structure from fixed-axis motion: Rigid structures. Biological Cybernetics, 54, 71-83.

Hoffman, D. D., Flinchbaugh, B. E. (1982). The interpretation of biological motion. Biological Cybernetics, 42, 195-204.

IsHIGUCHI, A. (1988). Interpolated elastic stnucture from the motion of dots. Perception \& Psychophysics, 43, 457-464.

Johansson, G. (1973). Visual perception of biological motion and a model for its analysis. Perception \& Psychophysics, 14, 201-211.

Johansson, G. (1975). Visual motion perception. Scientific American, 232, 76-88.

Pomerantz, J. R. (1983). The rubber pencil illusion. Perception \& Psychophysics, 33, 365-368.

TODD, J. T. (1982). Visual infomation about rigid and nonrigid motion: A geometric analysis. Joumal of Experimental Psychology: Human Perception \& Performance, 8, 238-252.

Tsai, R. Y., \& Huang, T. S. (1981). Uniqueness and estimation of three-dimensional motion parameters of rigid objects with curved surface (Coordinated Science Laboratory Report, R-921). University of Illinois at Urbana-Champaign.

UlLMAN, S. (1979). The interpretation of visual motion. Cambridge, MA: MIT Press.

ULLMAN, S. (1983). Recent computational studies in the interpretation of structure from motion. In J. Beck, B. Hope, \& A. Rosenfeld (Eds.), Human and machine vision (pp. 459-480). New York: Academic Press.

UlLMan, S. (1984). Maximizing rigidity: The incremental recovery of 3-D structure from rigid and nonrigid motion. Perception, 13, 255-274.

Vogels, R., \& Orban, G. A. (1985). The effect of practice on the oblique effect in line orientation judgments. Vision Research, 25, 1679-1687.

Wallach, H., \& O'ConNELl, D. N. (1953). The kinetic depth effect. Journal of Experimental Psychology, 45, 205-217.

(Manuscript received November 20, 1987; revision accepted for publication April 12, 1988.) 